\title{
Normal vascular and biliary hepatic anatomy: 3D demonstration by multidetector CT
}

\author{
P. Donato $\cdot$ P. Coelho $\cdot$ H. Rodrigues $\cdot$ E. Vigia $\cdot$ \\ J. Fernandes $\cdot$ F. Caseiro-Alves $\cdot$ A. Bernardes
}

Received: 1 September 2006 / Accepted: 18 June 2007 / Published online: 19 July 2007

(C) Springer-Verlag 2007

\begin{abstract}
Due to constant innovations in radiological and surgical techniques, more accurate results are expected in the diagnostic and therapeutic procedures related to hepatic pathology. The aim of this work was to demonstrate the normal hepatic vascular and biliary anatomy using cadaveric livers and CT scans of the affected livers. Furthermore, using the CT scans, the authors intended to illustrate the most common morphological variations of the vascular and biliary anatomy. Four human cadaveric livers were injected with colored silicone mixed with radiological contrast solution in the common bile duct, in the proper hepatic artery, in the portal vein and in the inferior vena cava near the ostia of the hepatic veins (only one of these structures was injected in each liver). After obtaining the CT scans, 3D rendered models were created, which demonstrated the normal hepatic anatomy of the vascular and biliary structures. The International Anatomical Nomenclature was used for their classification (based on Couinaud's work). The 3D rendered CT models were also modified to illustrate the most common normal variations of the hepatic anatomy (found in the literature).
\end{abstract}

P. Donato $\cdot$ H. Rodrigues $\cdot$ J. Fernandes $\cdot$ F. Caseiro-Alves

University Clinic of Radiology,

Coimbra University Hospital, Coimbra, Portugal

P. Coelho $(\square) \cdot$ A. Bernardes

Institute of Normal Anatomy,

Faculdade de Medicina da Universidade de Coimbra,

Coimbra Medical School, 3000 Coimbra, Portugal

e-mail: paulcoelho@netcabo.pt

E. Vigia

Curry Cabral Hospital, Lisbon, Portugal
Keywords Computed tomography x-ray $\cdot$ Bile ducts Liver anatomy $\cdot$ Liver segmentation · Portal vein . Imaging three-dimensional

\section{Introduction}

Hepatic surgery (especially hepatic resection) is based on the anatomical hepatic segmentation as described by Couinaud $[2,3]$.

There are several anatomical variations in the hepatic segmentation, which must be known, to perform an efficient surgical low-risk procedure. Some anatomical variations may demand the use of specific techniques or even contraindicate the surgical procedure [4].

Live donor hepatic transplants are increasingly frequent, due to the lack of compatible cadaveric donors [15]. Radiology plays an important role in the adequate selection of donors (preoperative CT is very important as it demonstrates the limits and volumes of the hepatic segments, and shows the hepatic vessels and bile ducts as well).

With this work, the authors aim, with the contribution of $\mathrm{CT}$, to create 3D models that represent the normal and main variations of the vascular and biliary anatomy of the liver. This will help hepatic radiologists and surgeons to review the most common hepatic anatomy, allowing the simultaneous visualization of the liver surface and inner vascular/ biliary anatomy in addition to providing the teachers with the material to be used in pedagogical activities.

\section{Materials and methods}

Four human cadaveric livers were injected with colored silicone mixed with radiological contrast solution in the 
common bile duct, in the proper hepatic artery, in the portal vein, and in the inferior vena cava near the hepatic veins ostia. Only one structure was injected in each liver and different colors were used in each one.

After being injected, the livers were scanned in the horizontal (axial) plane, using a double-scan $\mathrm{CT}\left(\mathrm{GE}^{\circledR}\right)$, a $1 \mathrm{~mm}$ collimation, with a pitch of 1.5 , and the images reconstructed with a $50 \%$ overlap.

The axial images were used to obtain 2D images and 3D rendered images using the surface shaded display algorithm. Two sets of contrast values were used: one to select the surface of the liver, and another, even higher, to select the contrasted structures. Both the images were combined, resulting in a 3D rendered model of the liver and its vascularization/biliary drainage.

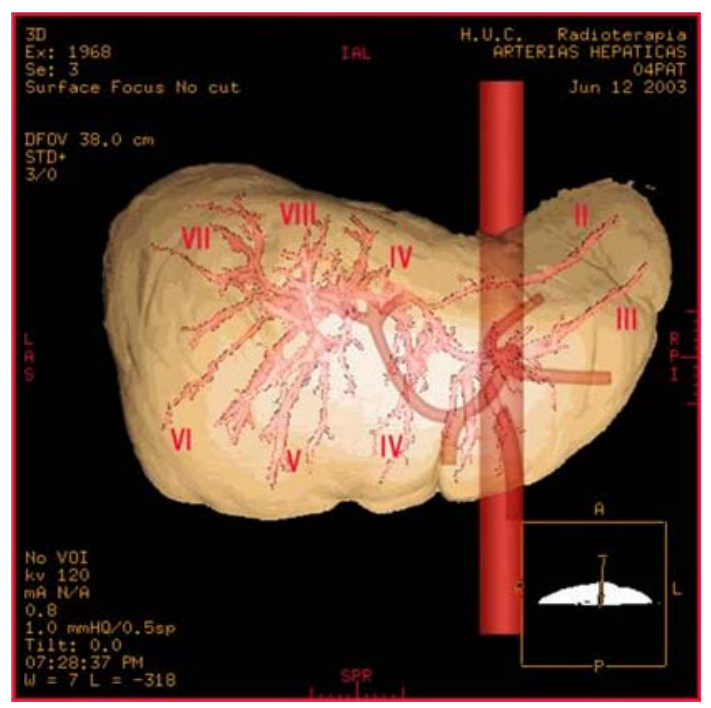

Fig. 1 Three-dimensional rendered model of a liver with the proper hepatic artery and its branches injected with silicone (the normal anatomy is shown)
Finally, using Adobe Photoshop 7.0 ${ }^{\circledR}$, the 3D rendered models were modified to illustrate the most frequent normal variations (described in the scientific literature).

\section{Results}

The normal hepatic anatomy and its most frequent variations can be demonstrated by the used technique.

The proper hepatic artery is one of the two terminal branches of the common hepatic artery, which is one of the branches of the coeliac trunk (Fig. 1). The common hepatic artery ends up in bifurcating into a right and left branch. The artery to segment IV (called intermediate branch or sometimes "middle hepatic artery") usually is a secondary branch of the left branch of the proper hepatic artery.

Michels (1955) divided the arterial vascularization in ten different patterns [13] (Table 1). The following variants were illustrated in this study: (1) the origin of the left branch of the proper hepatic artery from the left gastric artery with or without the persistence of its actual origin in $18 \%$ of the cases (Fig. 2); (2) the origin of the right branch of the proper hepatic artery from the superior mesenteric artery with or without the persistence of its actual origin in $18 \%$ of the cases (Fig. 3).

The normal portal vein ends up in a division into two terminal branches: the right and the left branch. The right branch of the portal vein receives the cystic vein before entering the liver, and after $1-3 \mathrm{~cm}$, it divides into an anterior branch (to segments V and VIII) and a posterior branch (to segments VI and VII). The left branch of the portal vein runs to the superior and posterior part of the left lobe of the liver (transverse part), bifurcating into two branches; a right one to segment IV and a left one to segment III (umbilical part). Segment I is vascularized independently, by the caudate branches that emerge from the portal vein or from one of its branches (Fig. 4).

Table 1 Proper hepatic arteries variations

\begin{tabular}{|c|c|c|c|c|c|c|}
\hline Type & $\begin{array}{l}\text { Prevalence } \\
(\%)\end{array}$ & $\begin{array}{l}\text { Right branch of the proper } \\
\text { hepatic artery origin }\end{array}$ & $\begin{array}{l}\text { Left branch of the proper } \\
\text { hepatic artery origin }\end{array}$ & $\begin{array}{l}\text { Accessory right } \\
\text { hepatic artery origin }\end{array}$ & $\begin{array}{l}\text { Accessory left } \\
\text { hepatic artery origin }\end{array}$ & Figures \\
\hline I & 55 & Proper hepatic artery & Proper hepatic artery & & & 1 \\
\hline II & 10 & Proper hepatic artery & Left gastric artery & & & 2 \\
\hline III & 11 & Superior mesenteric artery & Proper hepatic artery & & & 3 \\
\hline IV & 1 & Superior mesenteric artery & Left gastric artery & & & \\
\hline V & 8 & Proper hepatic artery & Proper hepatic artery & & Left gastric artery & 2 \\
\hline VI & 7 & Proper hepatic artery & Proper hepatic artery & Superior mesenteric artery & & 3 \\
\hline VII & 1 & Proper hepatic artery & Proper hepatic artery & Superior mesenteric artery & Left gastric artery & \\
\hline VIII & 2 & Superior mesenteric artery & Left gastric artery & Superior mesenteric artery & Left gastric artery & \\
\hline IX & 4.5 & Superior mesenteric artery & Superior mesenteric artery & & & \\
\hline $\mathrm{X}$ & 0.5 & Left gastric artery & Left gastric artery & & & \\
\hline
\end{tabular}




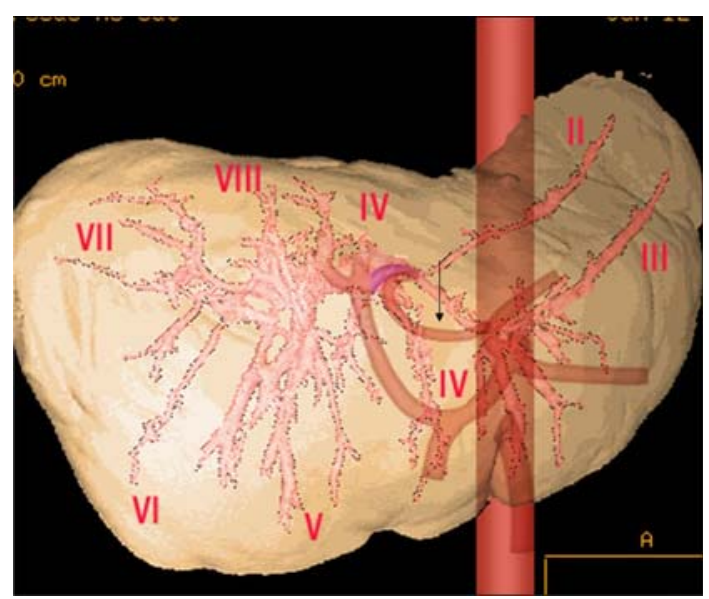

Fig. 2 Modified 3D rendered model of a liver with the proper hepatic artery and its branches injected. The left branch of the proper hepatic artery has its origin in the left gastric artery (arrow). The usual origin (from the proper hepatic artery may also be present)-Michels' type II and $V$-these variants have $18 \%$ prevalence

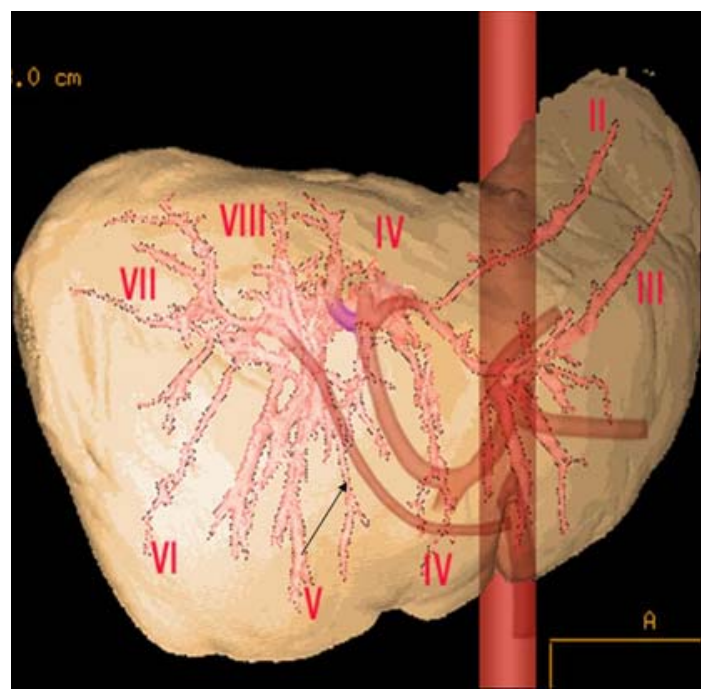

Fig. 3 Modified 3D rendered model of a liver with the proper hepatic artery and its branches injected. The right branch of the proper hepatic artery arises from the superior mesenteric artery (arrow). The usual origin may also be present-Michels' type $I I I$ and $V I$. The prevalence of this variant is $18 \%$

There are many variants related to the portal vein. The most frequent is the absence of the right branch of the portal vein, with the trifurcation of the vein into left, anterior and posterior branches, present in $10 \%$ of the population (Fig. 5).

Other variants were also highlighted in this work, namely the absence of a right branch of the portal vein, with the posterior branch (to segments VI and VII) being a collateral of the portal vein (Fig. 6), with a prevalence of $5 \%$ and the absence of the right branch of the portal vein

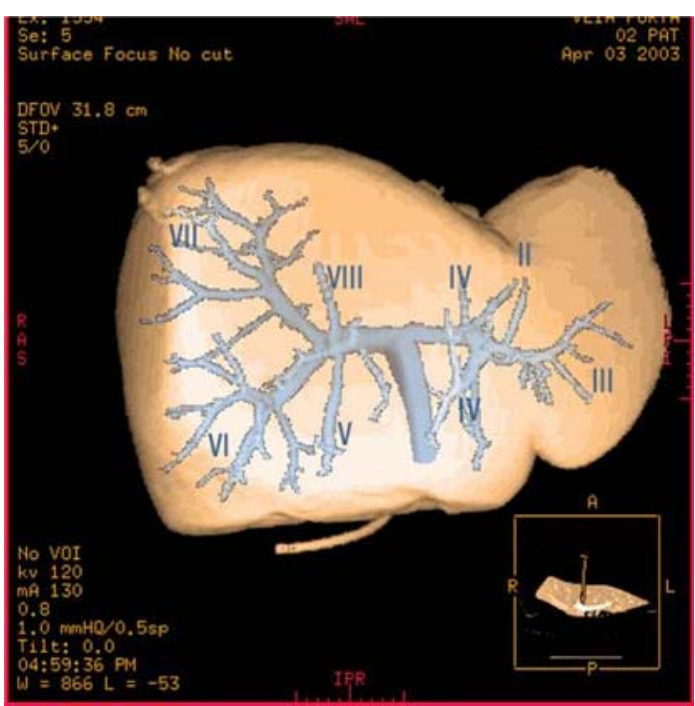

Fig. 4 Three-dimensional rendered model of a liver with the portal vein and its branches injected. The normal anatomy is shown. The right branch of the portal vein, after receiving the cystic vein divides into an anterior branch (to segments $V$ and $V I I I$ ) and a posterior branch (to segments $V I$ and $V I I)$. The left branch of the portal vein continues to segment $I I$ (transverse part), after giving a branch to segments $I I I$ and $I V$ (umbilical part)

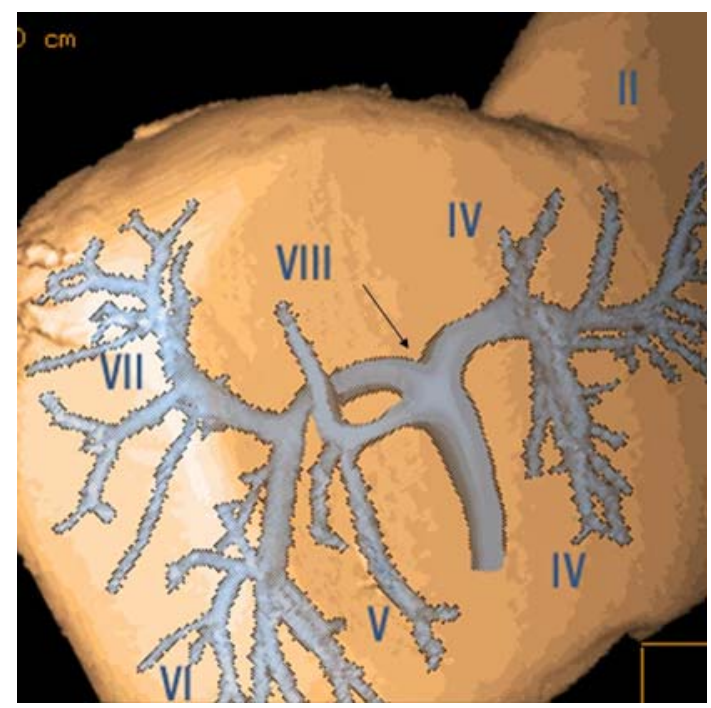

Fig. 5 Modified 3D rendered model of a liver with the portal vein and its branches injected. The arrow indicates the trifurcation of the portal vein into a left branch, an anterior branch (to segments $V$ and $V I I I$ ) and a posterior branch (to segments $V I$ and $V I I$ ) with the absence of the right branch of the portal vein

with the origin of the anterior branch (to segments $\mathrm{V}$ and VIII) from the left branch of the portal vein, in $2 \%$ of the cases (Fig. 7).

The common hepatic duct is formed by the union of the right and left hepatic ducts. The left hepatic duct is formed by the union of the ducts draining segment II (lateral branch) and segments III and IV (medial branch). It has a 


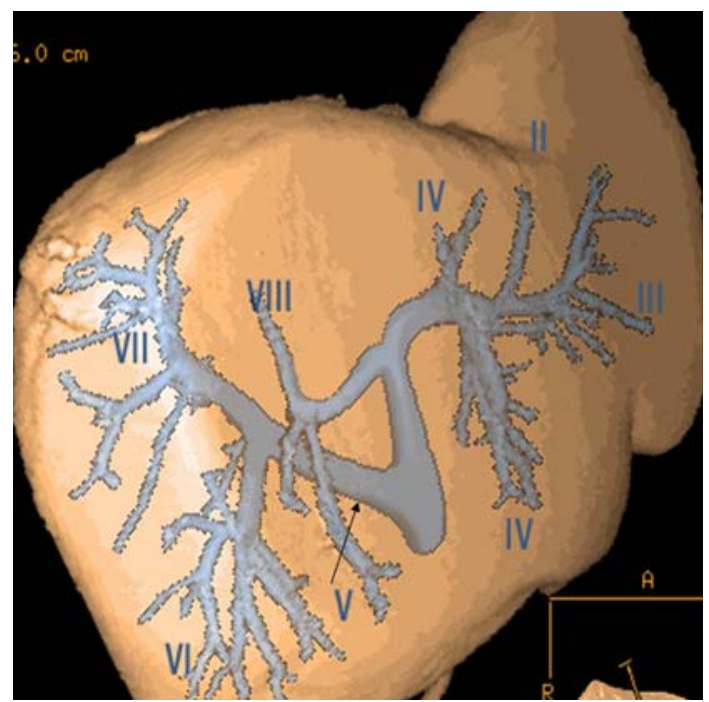

Fig. 6 Modified 3D rendered model of a liver with the portal vein and its branches injected. The portal vein gives a collateral posterior branch to segments VI and VII (arrow). The right branch of the portal vein does not exist. It is replaced by an anterior branch to segments $V$ and VIII

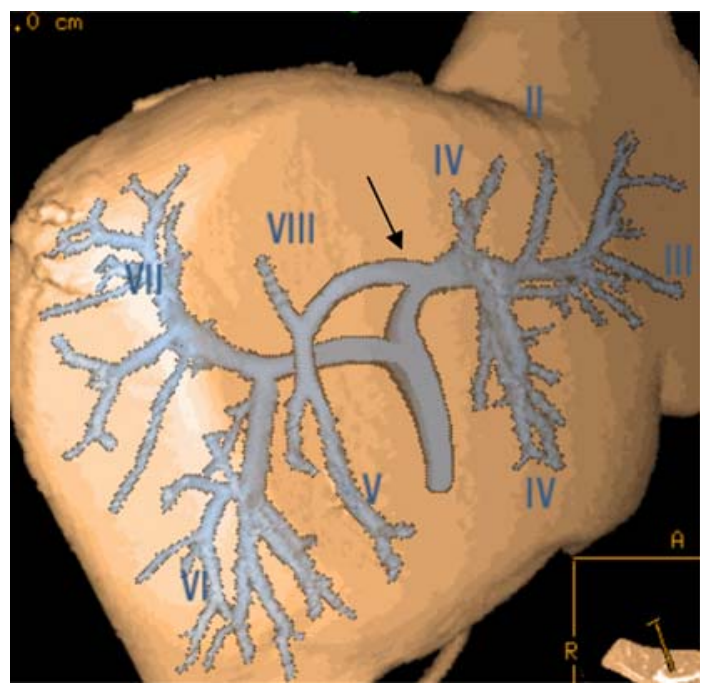

Fig. 7 Modified 3D rendered model of a liver with the portal vein and its branches injected. There is an absence of the right branch of the portal vein. The portal vein bifurcates into a left and posterior branch (to segments $V I$ and $V I I$ ). The anterior branch to segments $V$ and $V I I I$ arises from the left branch of the portal vein (arrow)

transversal orientation and a length of $1.5-3.5 \mathrm{~cm}$. The right hepatic duct is formed by the union of an anterior branch draining segments $\mathrm{V}$ and VIII and a posterior branch draining segments VI and VII. It is short, vertically orientated and has a length of $0.5-2.5 \mathrm{~cm}$ (Fig. 8).

The variants found were: (1) the drainage of the anterior branch (from segments V and VIII) directly into the common hepatic duct, with a prevalence of $16 \%$ in the population (Fig. 9); (2) the triple convergence of the anterior and posterior branches (from the right segments) and the left

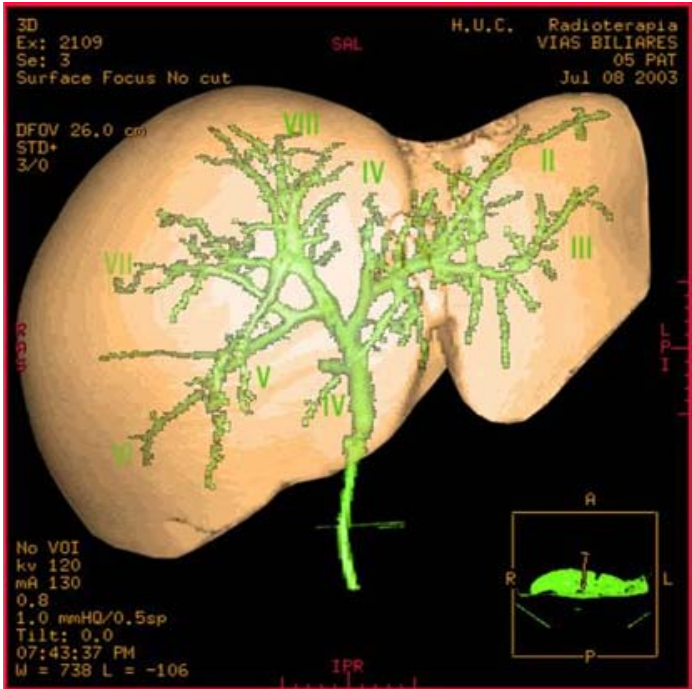

Fig. 8 Three-dimensional rendered model of a liver with the common bile duct and its branches injected. The left hepatic duct has a transverse orientation and it is formed by the lateral branch (from segment $I I$ ) and the medial branch (from segments $I I I$ and $I V$ ). The right hepatic duct is short and almost vertical and it is formed by an anterior branch (from segments $V$ and $V I I I$ ) and a posterior branch (from segments $V I$ and $V I I)$

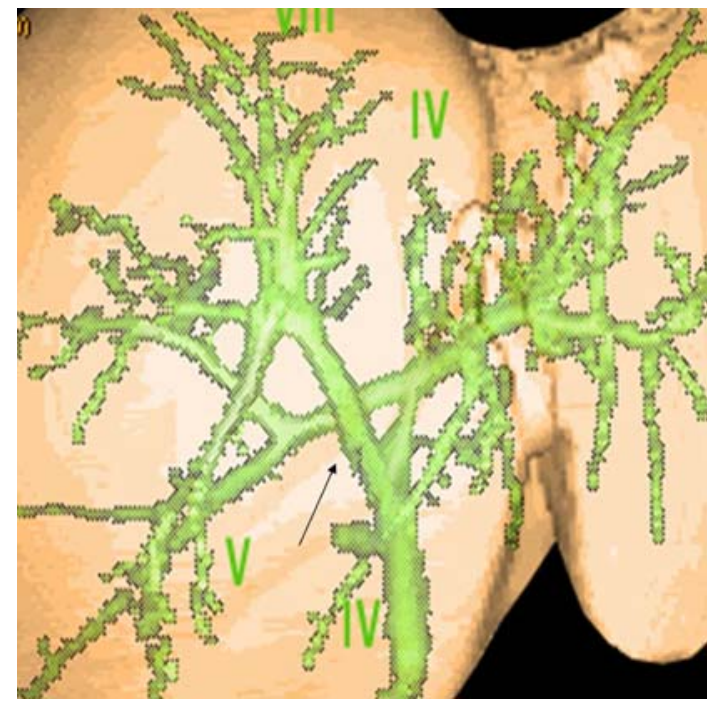

Fig. 9 Modified 3D rendered model of a liver with the common bile duct and its branches injected. The anterior branch of the right hepatic duct drains bile from segments $V$ and VIII directly to the common hepatic duct (arrow)

hepatic duct to form the common hepatic duct in $12 \%$ of the population (Fig. 10); (3) the drainage of the posterior branch (from segments VI and VII) directly into the common hepatic duct in $4 \%$ of the population (Fig. 11); and rarely, (4) the drainage of the duct from segment $\mathrm{V}$ directly into the gallbladder or the cystic duct (Fig. 12).

The right hepatic vein is dominant, but there is also a right accessory (inferior) hepatic vein. The middle and left 


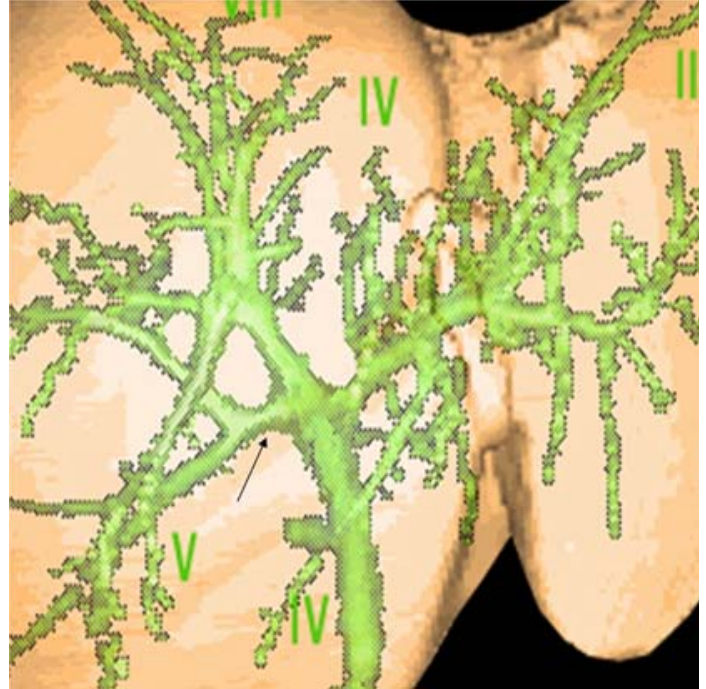

Fig. 10 Modified 3D rendered model of a liver with the common bile duct and its branches injected. The common hepatic duct is formed by the left hepatic duct and the anterior and posterior branches (from the right segments), with the absence of the right hepatic duct. The arrow shows the posterior duct from segments $V I$ and $V I I$

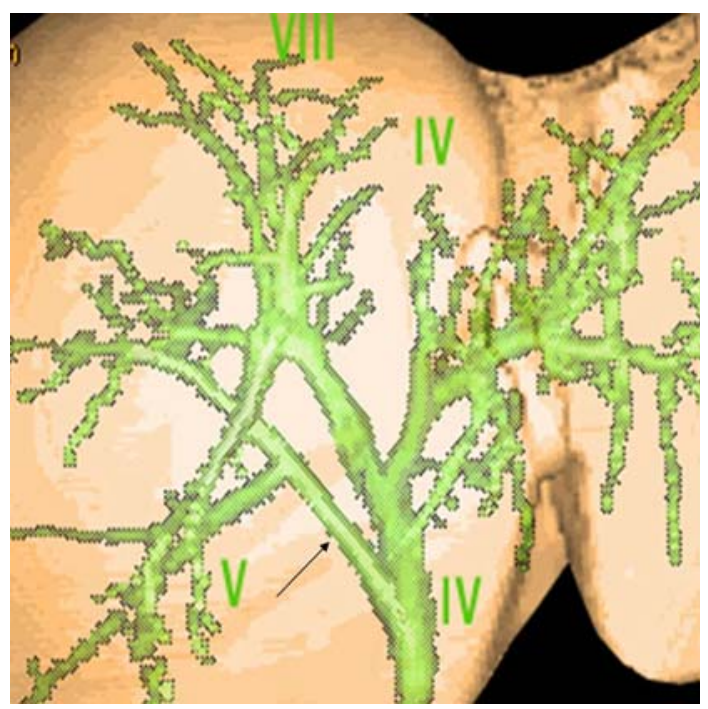

Fig. 11 Modified 3D rendered model of a liver with the common bile duct and its branches injected. The posterior branch from segments $V I$ and VII drains directly into the common hepatic duct (arrow)

hepatic veins form a common trunk that drains into the inferior vena cava. Segment I has its own venous drainage directly into the inferior vena cava. These small veins are called accessory hepatic veins (Fig. 13).

The most frequent variations found in literature were: (1) the right accessory hepatic vein is dominant and has a greater caliber than the right hepatic vein (Fig. 14); (2) the absence of the common trunk between the middle and the left hepatic veins (Fig. 15); and (3) the vein that drains the parenchyma near the falciform ligament is a tributary of the middle hepatic vein instead of the left hepatic vein.

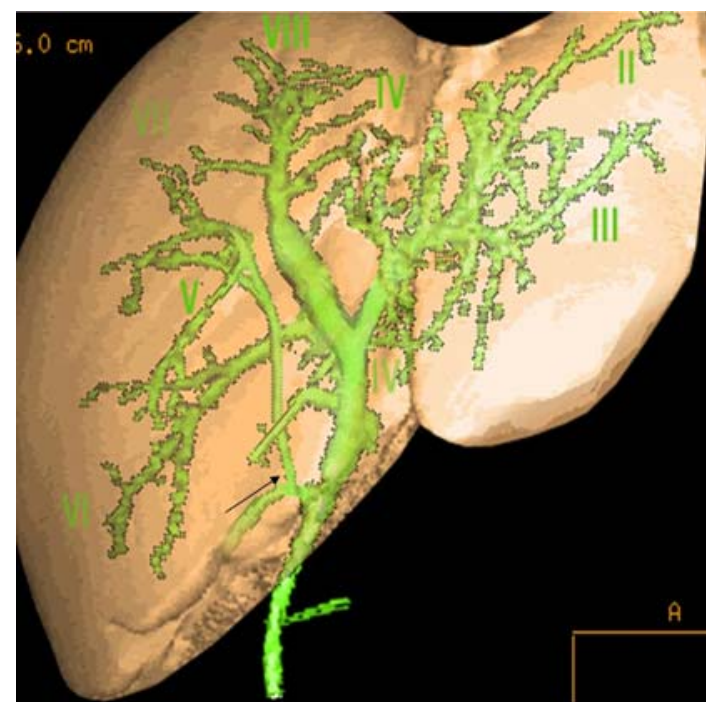

Fig. 12 Modified 3D rendered model of a liver with the common bile duct and its branches injected. The duct from segment $V$ (arrow) drains directly into the gallbladder or the cystic duct

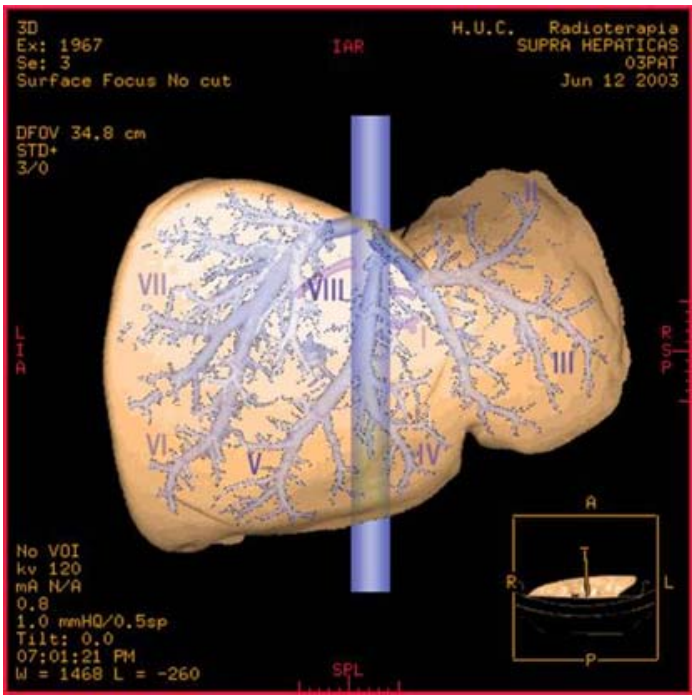

Fig. 13 Three-dimensional rendered model of a liver with the hepatic veins injected. The right hepatic vein is dominant, but an accessory (inferior) right hepatic vein also exists. The middle and left hepatic veins form a common trunk and drain together into the inferior vena cava. Segment I has is own drainage, directly into the inferior vena cava (accessory hepatic veins)

\section{Discussion}

The liver has a particular model of vascularization. Twentyfive percent of the hepatic blood flow is from the hepatic artery and the remaining $75 \%$ is from the portal vein $[7,16]$.

There are also other small arteries and veins that contribute with little significance to the hepatic blood flow, such as 


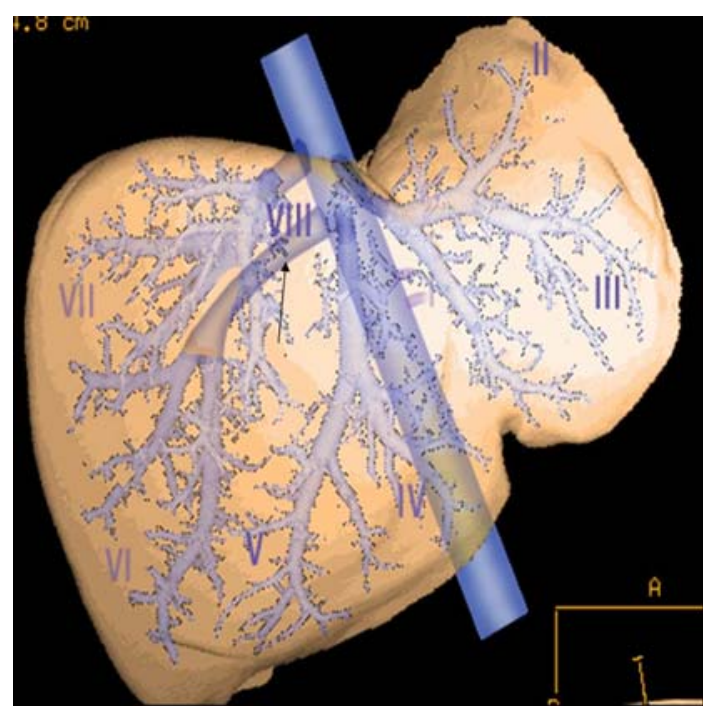

Fig. 14 Modified 3D rendered model of a liver with the hepatic veins injected. The accessory (inferior) right hepatic vein is dominant (arrow)

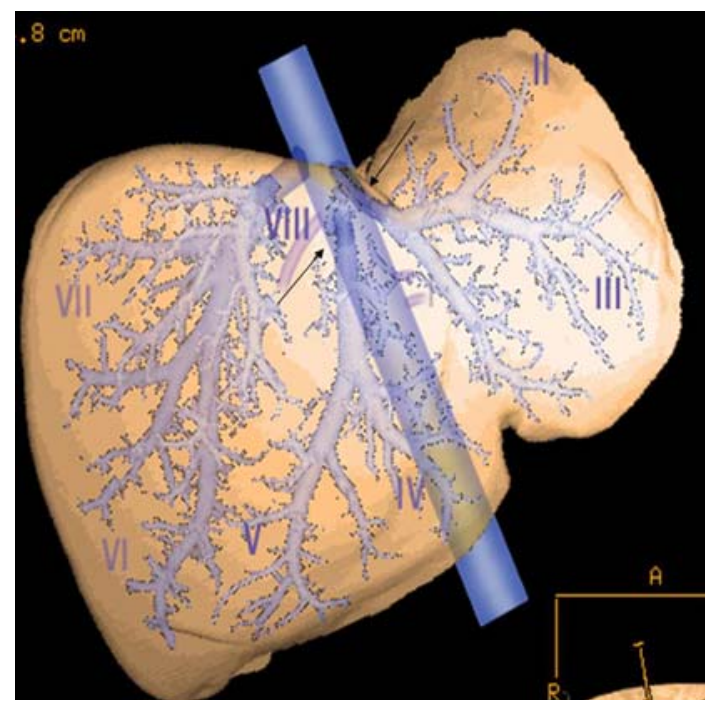

Fig. 15 Modified 3D rendered model of a liver with the hepatic veins injected. The middle and the left hepatic veins drain separately into the inferior vena cava (arrows)

the branches of the right inferior phrenic artery at areas near the falciform ligament, the cystic vein in segments IV and $\mathrm{V}$, the venous plexus near the gallbladder in a small portion of segment IV, the left gastric vein and the para-umbilical venous plexus that drains to the liver, blood from part of the anterior abdominal wall [16]. Although these vessels represent a small portion of the hepatic blood flow, it is important to know their existence, because they can be responsible for the presence of false lesions visible in CT scans, especially when using intravenous contrast (they can contrast earlier or later than the remaining parenchyma $[16,17])$. The steatotic liver can be heterogeneous, with spared areas. This is also explained by this particular vascularization, as the blood arriving to these areas has a different composition.

Modern surgical techniques, especially hepatic resection, are based on the anatomic hepatic segmentation as described by Couinaud. He divided the liver into eight segments based on the branching of the portal vein and the anatomy of the hepatic veins $[2,3]$.

There are many anatomical variants in the hepatic vascularization and biliary drainage that must be known by surgeons and radiologists so that an effective surgical procedure with low risk can be offered to the patient. It should be noted that some of the most common anatomical variants may even obligate different surgical techniques or even prohibit a surgical procedure.

During a partial hepatectomy, radiology allows the determination of the precise location of a lesion (determination of the involved segments), offers hepatic volumetric studies, facilitates the therapeutic embolization of vessels to change the hepatic volumetry, and moreover it assists in the diagnosis of anatomical variations $[4,12]$.

To avoid complications, the knowledge of anatomical variations is also important during biliary surgery [4].

Live donor hepatic transplant is a recent technique that is gaining importance due to the lack of compatible cadaveric livers [15]. Radiology plays a fundamental role in the selection of donors and planning of surgery.

Due to the risks presented to the live donors, a detailed preoperative study is mandatory. The hepatectomy dividing plane is the main portal fissure, which is relatively avascular and is located to the right of the falciform ligament, parallel to the middle hepatic vein, passing through the fossa for the gallbladder and the groove for the inferior vena cava (Cantile's line). To prevent the lesion of vessels, which may cause ischemia of the graft or of the remaining donor liver, it is of utmost importance to identify the vascular structures that cross this plane. It is also important to identify other anatomical variations in the vascular and biliary anatomy of the donor $[5,8,10]$.

The intermediate branch of the proper hepatic artery (the middle hepatic artery), which supplies segment IV, is usually a branch of the left branch of the proper hepatic artery, but it may also be a branch of the right branch of the proper hepatic artery and cross the surgical plane. In this case, if the right branch of the proper hepatic artery is ligated before the emergence of this artery, ischemia of segment IV may complicate the postoperative period.

The surgical procedure may also be difficult or even contraindicated if variations of portal branching, in the absence of the right branch of the portal vein, are present.

The documentation of the hepatic venous drainage is important to plan the number and localization of anastomoses that have to be conducted, thereby decreasing the operative time. For example, the most used technique of right lobe 
transplant preserves the donor middle hepatic vein. The presence of accessory hepatic veins or the drainage of the blood from segments V and VIII to the middle hepatic vein demands a higher number of anastomoses [5].

The knowledge of some vascular and biliary variations has clinically important implications and may even determine different therapeutic plans. The disrespect of these precautions may complicate procedures or even cause major complications [1, 4].

For example, during a left hepatectomy (removal of segments II, III, and IV), if there is an absence of the right branch of the portal vein and the anterior or posterior branch (draining segments V and VIII or VI and VII, respectively) emerges from the left branch of the portal vein (Figs. 6, 7), the ligation of the left branch of the portal vein may cause ischemia of the right segments vascularized by the branch arising from the left branch of the portal vein. In a right hepatectomy (removal of segments V, VI, VII, and VIII), if the anterior branch of the right hepatic duct (drains segments V and VIII) is a direct tributary of the common hepatic duct (Fig. 9) and the posterior branch of the right hepatic duct (drainage of segments VI and VII) is not identified, biliary collections may develop in those segments, during the postoperative period.

During a hepatectomy, the origin of the vessels to segment $\mathrm{I}$ is the decisive factor to remove or retain that segment, with the objective of avoiding its ischemia [4].

The origin of the intermediate branch of the proper hepatic artery (the "middle hepatic artery") from the proper hepatic artery or its left or right branches is also a factor to be considered, as it can be a cause of segment IV ischemia. For example, if it arises from the left branch of the proper hepatic artery, during a left hepatectomy (removing segments II and III), the left branch of the proper hepatic artery has to be ligated after the origin of the middle hepatic artery. In the case otherwise, however, ischemia of the segment IV will occur.

The knowledge and CT demonstration of the variations of the hepatic vascular and biliary anatomy are not the only important aspects. The identification of the normal vascular and biliary anatomy allows the radiologist to precisely locate the hepatic lesions with surgical indication $[1,6]$.

The knowledge of the vascular and biliary anatomy of the liver is of enormous importance if considering a live donor hepatic transplant surgical technique [4, 5]. For example, if the donor has multiple hepatic arteries, vascularising the hepatic lobes (dominants and accessories), the transplant may be contraindicated technically.

In a live donor hepatic transplant, the section plane is the main portal fissure, located to the right of the falciform ligament and parallel to the middle hepatic vein, distinguishing segments II, III, and IV, from segments V, VI, VII, and VIII [15]. A left hepatectomy is contraindicated when there is an absence of the right branch of the portal vein and the anterior branch arises from the left branch of the portal vein (Fig. 7), because it would cause ischemia of segments $\mathrm{V}$ and VIII $[4,15]$.

In a transplant with a donor right hepatectomy, the presence of variations of the portal vein, especially the most frequent (Figs. 5, 6,7) with the absence of the right branch of the portal vein, may also contraindicate the surgical procedure $[9,11,15]$. The origin of the intermediate branch of the proper hepatic artery (middle hepatic artery) also has to be identified, as the accidental ligation of this artery will cause ischemia of the segment IV [9, 15]. For example, if in a right hepatectomy this artery arises from the left branch of the proper hepatic artery (the most frequent situation), it may not cause any problem. However, contrarily, if the artery arises from the right branch of the proper hepatic artery, it may cause ischemia of segment IV.

The knowledge of the venous drainage is of utmost importance to determine the number and location of the venous anastomoses that have to be conducted $[5,9,14$, 15]. The most used technique in the right lobe transplant leaves the middle hepatic vein in the donor. The existence of accessory hepatic veins or other variations (for example when the branches from segments V and VIII drain to the middle hepatic vein) will demand a higher number of anastomoses to the host inferior vena cava. This knowledge before the surgery will allow a better planning and reduce the surgical time.

Even in more common surgeries, such as a cholecystectomy, the misdiagnosis of rare biliary variants as the anomalous drainage of segment $\mathrm{V}$ directly to the gallbladder or the cystic duct (Fig. 12) may result in the development of postoperative biliary collections in the fossa for the gallbladder [4].

\section{Conclusion}

The multidetector CT scans and the posterior 3D rendered images allow the demonstration of the hepatic anatomy, being useful for preoperative evaluations and pedagogical purposes.

The knowledge of anatomical variations is of the utmost importance in several clinical situations such as live donor hepatic transplant, partial hepatectomies and biliary surgery. These variations can be identified with the CT scans and the posterior $3 \mathrm{D}$ rendering of the images.

Acknowledgment Grants from the Investigation Support Cabinet, Coimbra Medical School.

\section{References}

1. Belli G, D'Agostino A, Ciciliano F, et al (2002) Liver resection for metastases: 15 years of experience. J Hepatobiliary Pancreat Surg 9:607-613 
2. Bismuth $H$ (1988) Surgical anatomy and anatomical surgery of the liver. In: Blumgart L (eds) Surgery of the liver and biliar tract. Churchill and Livingstone, Edinburg, pp 3-9

3. Couinaud C (1957) Le foie. Masson and Cie, Paris, p 530

4. Denys A, Sauvanet A, Wicky S, et al (2002) Anatomie chirurgicale du foie: ce qu'il faut savoir. J Radiol 83:205-218

5. Guiney MJ, Kruskal JB, Sosna J, et al (2003) Multi-detector row $\mathrm{CT}$ of relevant vascular anatomy of the surgical plane in split-liver transplantation. Radiology 229:401-407

6. Herfarth C, Lamade W, Fischer L, et al (2002) The effect of virtual reality and training on liver operation planning. Swiss Surg 8:6773

7. Itai Y, Matsui O (1997) Blood flow and liver imaging. Radiology 202:306-314

8. Kamel IR, Kruskal JB, Promfret EA, et al (2001) Impact of multidetector CT on donor selection and surgical planning before living adult right lobe transplantation. AJR Am J Roentgenol 176:193200

9. Kanazawa A, Hirohashi K, Tanaka H, et al (2002) Usefulness of three-dimensional computed tomography in a living-donor extended right lobe liver transplantation. Liver Transpl 8:10761079
10. Lee SS, Kim TK, Byun JH, et al (2003) Hepatic arteries in potencial donors for living related liver transplantation: evaluation with multi-detector row CT angiography. Radiology 227:391-399

11. Lo CM, Fan ST, Liu CL, et al (1997) Adult-to-adult living donor transplantation using extended right lobe grafts. Ann Surg 226:261-269

12. Madoff DC, Hicks ME, Vauthey JN, et al (2002) Transhepatic portal vein embolization: anatomy, indications, and technical considerations. Radiographics 5:1063-1076

13. Michels NA (1955) Blood supply and anatomy of the upper abdominal organs. Lippincott, Philadelphia, pp 1-581

14. Reichert PR, Renz JF, D'Albuquerque LAC, et al (2000) Surgical anatomy of the left lateral segment as applied to living-donor and split-liver transplantation. Ann Surg 5:658-664

15. Shiffman ML, Brown RS Jr, Olthoff KM, et al (2002) Living donor liver transplantation: summary of a conference at The National Institutes of Health. Liver Transpl 8:174-188

16. Vilgrain V, Vullierme MP, Djabbari M, et al (2002) Foie et anomalies de perfusion en imagerie. J Radiol 83:255-266

17. Yoshimitsu K, Honda H, Kuroiwa T, et al (2001) Unusual hemodynamics and pseudolesions of the noncirrhotic liver at $\mathrm{CT}$. Radiographics 21:S81-S96 